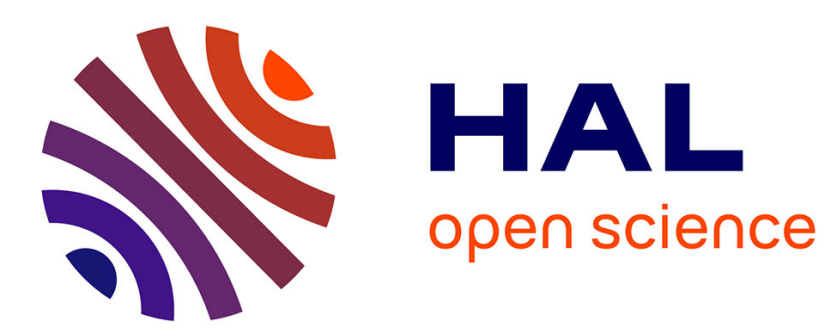

\title{
Sur la rationalité des rapports des moments magnétiques moléculaires et le magnéton
}

Pierre Weiss

\section{To cite this version:}

Pierre Weiss. Sur la rationalité des rapports des moments magnétiques moléculaires et le magnéton. Radium (Paris), 1911, 8 (8), pp.301-307. 10.1051/radium:0191100808030101 . jpa-00242486

\section{HAL Id: jpa-00242486 https://hal.science/jpa-00242486}

Submitted on 1 Jan 1911

HAL is a multi-disciplinary open access archive for the deposit and dissemination of scientific research documents, whether they are published or not. The documents may come from teaching and research institutions in France or abroad, or from public or private research centers.
L'archive ouverte pluridisciplinaire HAL, est destinée au dépôt et à la diffusion de documents scientifiques de niveau recherche, publiés ou non, émanant des établissements d'enseignement et de recherche français ou étrangers, des laboratoires publics ou privés. 


\section{Sur la rationalité des rapports des moments magnétiques moléculaires et le magnéton ${ }^{1}$ \\ Par Pierre WEISS \\ [Universili de Zurich - Lahoratoire de Physique.]}

M. Weiss se propose dans le présent mémoire de montrer " comment on peut trouver en valeur absolue le moment magnétique d'un grand nombre de molécules, et que tous ces moments ont une même valeur aliquote commune ») à laquelle il a donné le nom de " magnéton ».

Rèsumé de la théorie cinétique du magnétisme. Théorie de M. Langevin. - A la base de toute cette théorie se trouve un mémoire de M. Langevin ${ }^{2}$, dont nous rappellerons d'abord les points essentiels. Chaque molécule contiendrait un grand nombre d'électrons décrivant des orbites fermées, chacun d'eux fournissant ainsi un cerlain moment magnétique. Si, dans une matière constituée par de telles molécules, on établit un champ magnétique, les électrons modifient leur mouvement de manière à s'opposer à la création du champ magnétique; ce phénomène constitue le diamagnétisme; il est aussi général que le phénomène de Zeemann, auquel il cst intimement lié; chaque atome y participe avec une intensité qui lui est propre, et qui est à peu près indépendante des conditions plıysiques ou chimiques dans lesquelles il se trouve.

Lc paramagnétisme est un phénomène essentiellemont différent que, seules, présentent les molécules dont le moment magnétique résultant est différent de zćro. Sous l'action du champ magnétique extérieur, culles-ci tendent à s'orienter de manière que leurs axes magnétiques soient parallèles au champ extéricur et de même sens que lui. M. Langevin admet

1. Ellail d'un Hémoire général communiqué par lauteur. 2. Langun. Ann. Chim. Phys. 5 (1905) 70. que l'action de ce champ extérieur sur l'oricn'acion des molécules se fait suivant la mème loi que l'action de la pesanteur sur la répartition des molécules d'air dans l'almosphìre terrestre; il est ainsi conduit à l'équalion :

$$
\frac{\sigma_{m}}{\sigma_{m s}}=\operatorname{co}: \operatorname{li} a-\frac{1}{a}
$$

dins laque'le :

$$
a=\frac{\sigma_{m o} \mathrm{H}}{\mathrm{RT}}
$$

$\sigma_{m}$ est l'aimantation moléculaire (moment magnétique de la molécule-gramme);

II, est le champ magnétique;

$\mathrm{T}$, la température absolue;

R, la constante des gaz parfaits relative à la moléculegramme $\left(R=83,155.10^{6} \mathrm{er}_{0}^{r} \mathrm{~s}-\right.$ degrés $)$; c'est iussi

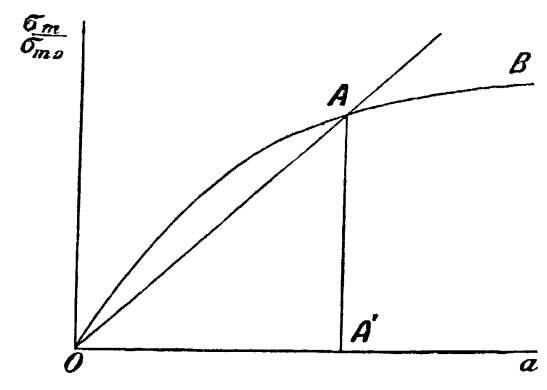

Fig. 1.

la force vive d'une moléculc-gramme par degré de liberté ;

Enfin $\sigma_{\text {Ino }}$ est une constante : le deuxième membre de 
l'équation I tendant vers l'unité lorsque $a$ devient infini, $\sigma_{m o}$ représente donc la valeur limite de l'aimantation $\sigma_{m}$ lorsque $H$ derient infiniment grand, ou bien $\mathrm{T}$ infiniment petit.

L'équation (1) est représentée graphiquencnt par la courbe (fig. 1) de la page 501 .

Si l'on ne considire que des petites valeurs $a$, on peut dérelopper le 2 membre del'équation 1 en séric, et n'en conserver que le premier terme. Il vientalors :

$$
\frac{\sigma_{m}}{\sigma_{m o}}=\frac{a}{3}=\frac{\sigma_{m o} \mathrm{H}}{3 \mathrm{li} T}
$$

or $\frac{\sigma_{m}}{\text { II }}$ est le coefficient d'aimantation moléculaire, que l'on désigne par \% on a donc

$$
\%=\frac{\sigma_{m 0}{ }^{2}}{31, T}
$$

Un retrouve ainsi la loi que $P$. Curie avait démontrée expérimentalement. On peut encore l'écrire:

$$
\%_{m} \mathrm{~T}=\frac{\sigma_{m o}{ }^{2}}{3 \mathrm{l}}=\mathrm{C}_{m}
$$

$\mathrm{C}_{m}$ ćtant la constante molículaire de Curie.

Théorie du champ moléculaire. - La lhéuric de M. Langevin convient parfaitemsnt aux gaz magnétiques, tels que l'oxygène, dans lesquils les molécules peuvent être considérées comme absolument libres, en dehors de leurs chocs; mais elle ne rend pas compte de nombreux phénomènes présentés par les corps magnétiques solides, dans lesquels interriennent les actions intermoléculaires. Pour tenir compte de ces dernières M. P. Weiss a imaginé l'hypothèse du champ moléculaire: " l'aclion de l'ensemble des molécules sur l'une d'elles serait équivalente à un champ magnétique uniforme, proportionnel à l'intensité d'aimantation et dirigé comme clle »). Cette hypothèse se traduit par l'équation :

$$
\mathrm{H}_{m}=\mathrm{NI}
$$

$\mathrm{II}_{m}$ étant le champ moléculaire.

I, l'aimantation (moment magnélique par unité de volume);

$\mathrm{X}$, une constante.

Si l'on exprime I en fonction de $\sigma_{m}$, on a encore :

$$
\mathrm{II}_{m}=-\mathrm{N} \frac{\mathrm{l}}{m} \sigma_{m}
$$

I) étant la densité du corps magnétique; $m$, son poids moléculaire.

M. Weiss admet encore « que ces actions mutuelles, exprimées par l'équation (6) sont les seules qui interviennent dans la rotation des molécules, ou, en d'autres termes, qu'à part le clıamp moléculaire, celles-ci tournent aussi librement dans un mélal magnélique, que dans un gaz parfait ").

On est ainsi conduit à admettre l'existence d'une aimantation spontanée dans les corps ferro-magnétiques, en l'absence de tout champ extérieur; et l'on peut des équations (1) et (7) déduire une valeur de cclle-ci. En effet, à une valeur $\mathrm{H}_{m}$ du champ moléculaire il corres,ond une valeur de a donnée par :

$$
a=\frac{\left.\sigma_{m \theta} \mathrm{NI}\right)}{m \mathrm{KT}} \sigma_{m},
$$

équation représentée sur la fig. (1) par la droite 0.1. Or $\sigma_{m}$ est déjà relié à $a$ par l'équation (1); il en résulte que les seules valeurs possibles, pour l'aimantation spontanée, sont déterminées par les deux points d’inter:ection 0 et A de la droite $0 \mathrm{~d}$ arec la courbe (1). Or de ceux-ci, scul $\Lambda$ correspond à un état stable, et peut par suite ètre accepté : son ordonnée donne done l'aimantation spontanće.

On peut encore, au mojen du diagramme (I) suivre la variation de l'ilimantation spontanée en fonction de la température : en effet, le coefficient angulaire de la droite (8) est proportionnel à T; le point A se rapproche done de l'origine lorsque ' $\mathrm{T}$ augmente, pour parrenir à celle-ci lorsque la droiie 0.1 derient tangente à la courbe de Iangevin; la va!cur $\Theta$ que prend alors $\mathrm{T}$ est la température, a': solue de perte du ferro-magnétisme spontané, que M. Weiss a désignéc sous le nom de point de Curie.

Lorsque $\mathrm{T}$ est voisin de $\theta$, l'aimantation sfontanće $\sigma_{m}$ et par suite $a$ sont petits, et l'on peut se servir de l’équation (3), qui, combinée à (2), donne :

$$
1=\frac{\left.\sigma_{m v^{2}} \mathrm{NI}\right)}{\delta m \mathrm{R}(\theta)}
$$

soil

$$
\Theta=\frac{\left.\sigma_{m v^{2}} \mathrm{Nl}\right)}{5 m \mathrm{~K}}
$$

soit encore, en introduisant $\mathrm{C}_{m}$ :

$$
\left.\theta=\frac{\mathrm{C}_{m}}{m} \mathrm{NI}\right)
$$

ou bii 11

$$
\Theta=\text { CNII }
$$

en désignant par G la constante de Curie rapportic à l'unité de masse.

Celte dernière relation est intéressante en ce qu'elle exprime $\mathrm{N}$ au moyen de grandeurs accessibles à l'expérience.

En divisant (8) par (9) il vient :

$$
\frac{\mathrm{T}}{\mathrm{\Theta}}=\frac{5}{a} \frac{\sigma_{m}}{\sigma_{m o}}
$$

el celte éfuation, jointe à (1) :

$$
\frac{\sigma_{t: l}}{\sigma_{m a}}=\operatorname{coth} a-\frac{1}{a}
$$


permet de définir des états correspondants des corps magnétiques, en les caractórisant par les valeurs de $\frac{\mathrm{T}}{(m)}$ et $\frac{\sigma_{m}}{\sigma_{m 0}}$.

La figure (2) porte, outre la courbe théorique tracéc en trait plein, les points obtenus expérimentalement pour la magnélite: on voit qüil y a une concordance très satisfaisante entre la théoric el l'expérience, saul

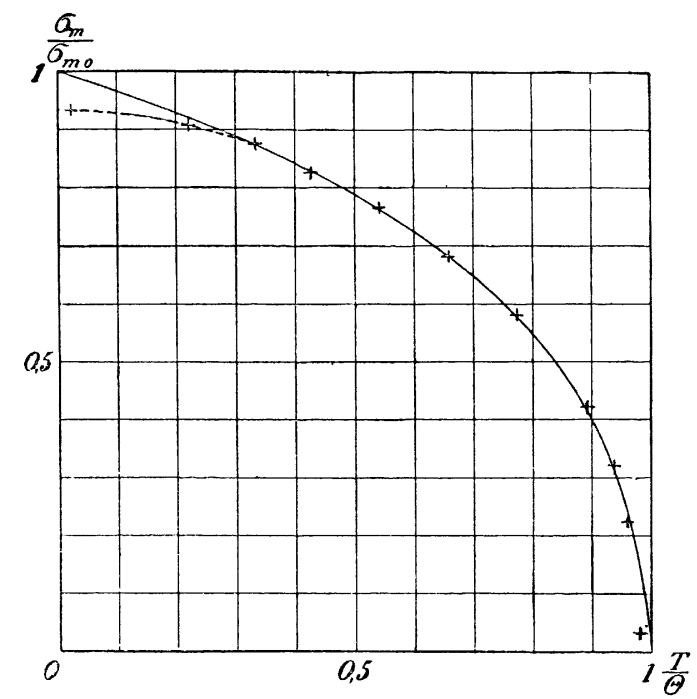

Fig. 2.

aux environs clu zéru absolu et du point de Curie. L'alliage $\mathrm{Fe} \mathrm{Ni}$ vérifie la loi théorique dans tout l’intervalle de 0 à $\Theta$; le fer, le nickel, le cobalt, par contre présentent avec elle des divergences notables.

Supposons maintenant qu'il y ait un champ extérieur $\mathrm{H}_{e}$; alors le champ magnétique tolal II sera :

$$
\mathrm{II}=\mathrm{II}_{e}+\mathrm{N} \text { I. }
$$

Supposons donnće la valeur de $\frac{\sigma_{m}}{\sigma_{m o}}$ et par suite la valeur de $a$; soit T' la température qui lui correspond cu l'alssence de tout champ extérieur, et 'l' la température (plus grande que T) qui correspond à cette mème valeur de $a$ lorsque le champ extérieur est II L’équation (8) donne :

$$
a \mathrm{RT}=\sigma_{m} \sigma_{m o} \mathrm{~N} \frac{\mathrm{D}}{m}
$$

puis, en combinant $(1$ 1) et $(2)$ on a :

$$
a \mathrm{R} \mathrm{T}=\tau_{m o} \mathrm{II}_{\iota}+\sigma_{m o} \sigma_{m l} \mathrm{~N} \frac{\mathrm{D}}{m \iota}
$$

d'ò

$$
a \mathrm{R}\left(\mathrm{T}^{\prime}-\mathrm{T}\right)=\sigma_{m}, \mathrm{IJ}_{e}
$$

Si $\tau_{m}$ est petit, on a vu que T' est voisin de $\Theta$, de sorte qu'on peut écrire :

$$
\left.\frac{\sigma_{m}}{\overline{\mathrm{I}}_{e}}\left(\mathrm{~T}^{\prime}-\because\right)^{\prime}\right)=\frac{\sigma_{m{ }^{2}}{ }^{2}}{5 \mathrm{~K}}=\mathrm{C}_{m}
$$

soit

$$
\%^{\prime \prime}(\mathrm{I}-\Theta)=\mathrm{C}_{m} \text {. }
$$

Pour les corps ferromagnétiques, cette dernière équation est à l'équation de Curie (5), ce que la loi de van der Waals est à la loi de Mariotte pour les gaz. Elic montre comment on peut obtenir la constante de Curie, par des expériences faites au-dessus de la température $\Theta$. II. Weiss a montré qu'elle est confirmée par les résultats numériques qui figurent dans le travail de $P$. Curie; il l'a lui-même rérifiée récemment sur plusieurs corps ferro-magnétiques, au cours d'un travail avec la collaboration de I. Foex.

On est ainsi amené à distinguer pour les métaux magnétiques les cas suivants :

$1^{\circ}$ Le ferro-magnétisme spontanć du zéro absolu au point de Curie;

$2^{\circ}$ Le ferromagnétisme sollicité, caractérisé par la formule (17);

$3^{\circ}$ Le paramagnélisme pur, caractérisé par un coclficient d'aimantation proportionuel a $\frac{1}{\mathrm{~T}}$.

En deliors de celle énuméralion, il faut signaler les cas de magnétisme faible, observés par du Bo's et Ilouda, où \% est constant, ou augmente arec T'.

Chaleur spécifique et champ moléculaire. L'hypothèse du champ mo'éculaire donne une interprétation de la variation brusque que subit à la température $\Theta$ la chaleur spécifique des corps ferro-magnétiques. Elle conduit, en effet, à ajouter, à ce que serait la chaleur spécifique d'un tel corps s’il n'était pas magnétiqus, un terme

$$
c^{\prime}=+\frac{1}{\mathrm{~J}} \frac{\mathrm{dE}}{\mathrm{at}}
$$

J étant l'équivalent;

E, l'énergie d'aimantation rapportée à l'unité de masse.

Ce terme, qui peut être déterminé par des expériences purtment magnétiques, devient brusquement nul à la température $\Theta$. Il en résulte une cessation brusque de la chaleur de désaimantation qu'il reprísente, d'où la discontinuité observée. Conformément à cette manière de voir, MM. Weiss et Beck ont obtenu des valeurs de $\Theta$ concordantes pour différentes substance:, en employant soit la méthode magnétique, soit la méthode calorimétrique.

Le Magneton. - Cette théorie, qui est confirméc d'une manière si frappante par les phénomènes généraux du ferromagnétisme, échoue cependant lorsqu'il s'agit d'expliquer certaines propriétés de substances ferro-magnétiques particulières: c'est le cas, par exemple, de la courbe exprimant $\frac{1}{\%}$ en fonction de T audessus du point de Curie pour la magnélite; au lieu d'une seule droite, donnée parl'équation (14), nous avons ici loute une série de droites se suirant après des 
condes brusques, ou une courbe de raccordement (Voir fig. 5). On peut se demander si l'on n'interpréterait pas ces particularités, en assujettissant à varièr suirant

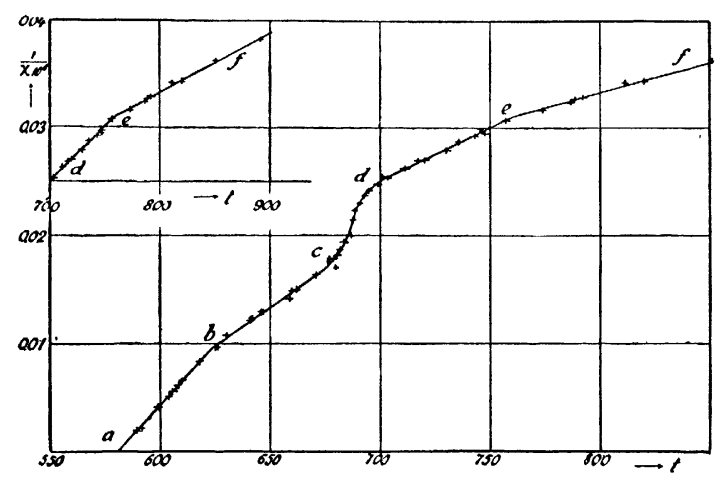

Fig. $\bar{~}$.

des lois convenables les grandeurs qui jusque-là ćtaient supposées constantes pour un même corps ferro-magnétique, à savoir $\mathrm{N}, m, \sigma_{m o}$.

M. Weiss chercha d'abord, mais sans obtenir de bons résultats, à rendre variable la constante $\mathrm{N}$; il pensa ensuite à $m$ : les coudes de la courbe de la magnétite correspondraient alors à des chạngements d'états, à des polýmérisations. A chaque portion rectiligne ds la courbe il correspond une constante de Curic $\mathrm{C}_{m}$ distincie, que l'on peut déduire de l'inclinaison de cette portion au moyen de l'équ. (17). On a ensuite :

$\mathrm{C}_{m}=\frac{\sigma_{m o}{ }^{2}}{5 \mathrm{R}} \quad$ (5) ou bien $\quad \mathrm{G}=m \frac{\sigma_{0}{ }^{2}}{3 \mathrm{R}} \quad$ (j) bis.

C et $\sigma_{0}$ étant les constantes rapportées à l'unité de masse.

N. Weiss admet que les atomes conservent des moments magnétiques constants, par suite que $\sigma_{0}$ est constant dans tout l'interville des températures. L'équation (o bis) permettra donc de calculer le degré de polymérisation correspondant à chaque portion recliligne de la courbe. Or il se trouva que les valeurs de $m$ (en prenant arhitrairement $m=1$ pour la portion rectiligne relative aux températures les plus basses), ne sont que trìs grossièrement des nombres entiers; de plus $m$ irait en cro.ssant lorsque la température augmente, ce qui est contradictoire avec les faits les plus habituels de la chimie.

Si l'on rejette l'hypothìse de la variabilité de $m$, il ne reste donc plus qu'à envisager des variations de $\sigma_{m o}$. Cette dernière manière de voir conduit, en ce qui concerne cette même courbe de la magnétite, aux résultats inscrits dans le tableau I.

Les nombres écrits sous les accolades marquées $\mathrm{F}$ et $\mathrm{W}$ ont été respectivement calculés par $\mathrm{M}$. Foex et M. Weiss en partant de la mème courbe expérimentale. (fig. 5).

Il est facile de reconnaitre dans ce tableau, qu'avec
Tableau I.

\begin{tabular}{|c|c|c|c|c|c|}
\hline $\begin{array}{c}\text { Intervalle } \\
\text { de } \\
\text { lempérature }\end{array}$ & C & $\sigma_{0}$ & C. & $\sigma_{0}$ & 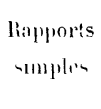 \\
\hline $\begin{array}{c}581^{\circ}-622^{\circ} \\
622^{\circ}-680^{\circ} \\
710^{\circ}-770^{\circ} \\
7.0^{\circ}-900^{\circ} \\
>900^{\circ} \text { Curie) }\end{array}$ & $\mid \begin{array}{l}0,0444 \\
0,0682 \\
0,010 \vdots \\
1,0180 \\
0,028\end{array}$ & $\begin{array}{l}1 \\
1,258 \\
1,535 \\
2,013 \\
2,515\end{array}$ & $\begin{array}{l}0,0445 \\
0,0663 \\
0,01025 \\
0,01776 \\
0,028\end{array}$ & $\begin{array}{l}1 \\
1,22 \\
1,513 \\
1,995 \\
2,515\end{array}$ & $\begin{array}{l}1 \\
1,25 \\
1,5 \\
2 \\
2,5\end{array}$ \\
\hline
\end{tabular}

une grande précision, les grandeurs $\sigma_{0}$ sont entre elles comme les nombres $4,5,6,8,10$.

C'est là un premier argument en faveur de l'hypothèse énoncée au début de cet extrait, et d'après laquelle les moments magnétiques des molécules seraient des multip!es entiers d'un mème moment magnétique élémentaire, le magnéton. Remettant à plus loin une vérification plus générale de cette thèse, nous dirons de suite avec M. Weiss comment ce magnéton peut être déterminé en valeur absolue, au mojen des expériences qu'il a fait's à Leyde avec la collaboration de I. Kamerlingh Onne:, afin de déterminer l'aimantation de diverses substances aux très basses tempéralures. Les rósultats obtenus sont reproduits dans le tableau ci-dessous, qui donne l's moments magnétiques del'atome-gramme et de $\frac{1}{3}$ de la molécule gramme.

$$
\begin{array}{lrrr}
\text { Nickel . . . . . . . . . } & 5,581 \\
\text { Cobalt . . . . . . . . . } & 9,650 \\
\text { Fer . . . . . . . . . . . } & \mathbf{1 9 , 4 1 0} \\
\text { Magnétite . . . . . . . . } & 7, \mathbf{7 1 7}
\end{array}
$$

Les nombres relatifs au cobalt et à la magnélite sont incertains; mais ceux du nickel et du fer, sur lesquels on peut compter, sont dans le rapport de 11 a 5 . $\Lambda$ près les corrections dues à la dilatation thermique, on trouve pour partie aliquote commune à ces 2 nombres :

$$
\begin{gathered}
\text { Pour le fer } 12560: 11=\begin{array}{c}
1125,6 \\
\text { Pour le nickel } 3,570: 5= \\
\text { Moyenne }
\end{array} \frac{112 \overline{5,5}}{1125,5}
\end{gathered}
$$

Cette partie aliquote est appe'će magnéton-gramme; M. Weiss l'estime exacto à deux ou trois millièmes près. En la divisant par le nombre d'atomes dans l'atome-gramme, soit $68,5.10^{22}$, on trouve

$$
16,40 \times 10^{-22}
$$

pour le moment magnétique de l'aimant élémentaire, le magnéton lui-même.

Une confirmation remarquable est apportée à celle théorie par l'examen des résultats numériques obtenus séparément par différents auteurs.

Corps magnétique en solution. - M. Weiss rappelle en premier licu le mémoire de Pascal ${ }^{1}$ sur 1. Paccal, Ann. Chim. P'ilys., 16 (1909) 551. 
l'aimantation des solutions étendues de sels paramagnétiques. A de telles solutions la théorie primitive de II. Langevin s'applique complètement ; on peut donc se servir de l'équation

$$
\sigma_{m o}^{2}=3 \mathrm{RC}_{m}
$$

pour déterminer $\sigma_{m o}, \mathrm{C}_{m}$ étant connu grâce à la loi de Curic, dès que l'on a déterminé le coefficient d'aimantation $\%_{m}$ à une température connue.

Il est nécessaire de préciser ici, "que la molécule magnélique est la quantité de matière dont l'axe magnétique possède deux degrés de libertés d'orientation; pour fixer les idécs, si plusieurs atomes d'une mime molécule chimique sont reliés entre eux par des articulations ayant la mobilité d'un genou de Cardın, chacun d'eux doit être considéré comme une molécule magnétique distincte. On suppose dans la suile que chaque molécule ne contient quiun atome magnétique, c'est-à-dire que là où la formule chimique en indique plusieurs, ils sont articulés entre eux »).

Les résultats de Pascal ont permis à $\mathrm{M}$. Weiss de dresser le tableau II.

Nous rappellerons que les nombres $\%$ ont été déterminés par Pascal, en prenant pour l'eau $\%_{m}=-0,75.10^{-6}$; ils comportent une correction due au diamagnétisme des divers.atomes entrant dans les molécules considérées. On voit sur ce tableau que, sur 27 corps étudiés, il y en a 17 pour lesquels l'accord arec la théorie est excellent; il n'y a même de désaccord absolu que pour le ferro-cyanure de potassium et les deux derniers sels de vanadium.

En calculant la valeur des magnétons-grammes, d'après les nombres fournis par les corps marqués 2 , $5 ; 4 ; 5,6,10,11 ; 7 ; \mathrm{M}$. Weiss a trouvé le nombre 1122,1 qui ne diffère que de $\frac{1,5}{1000}$ de la valeur donnéc

Tableau II.

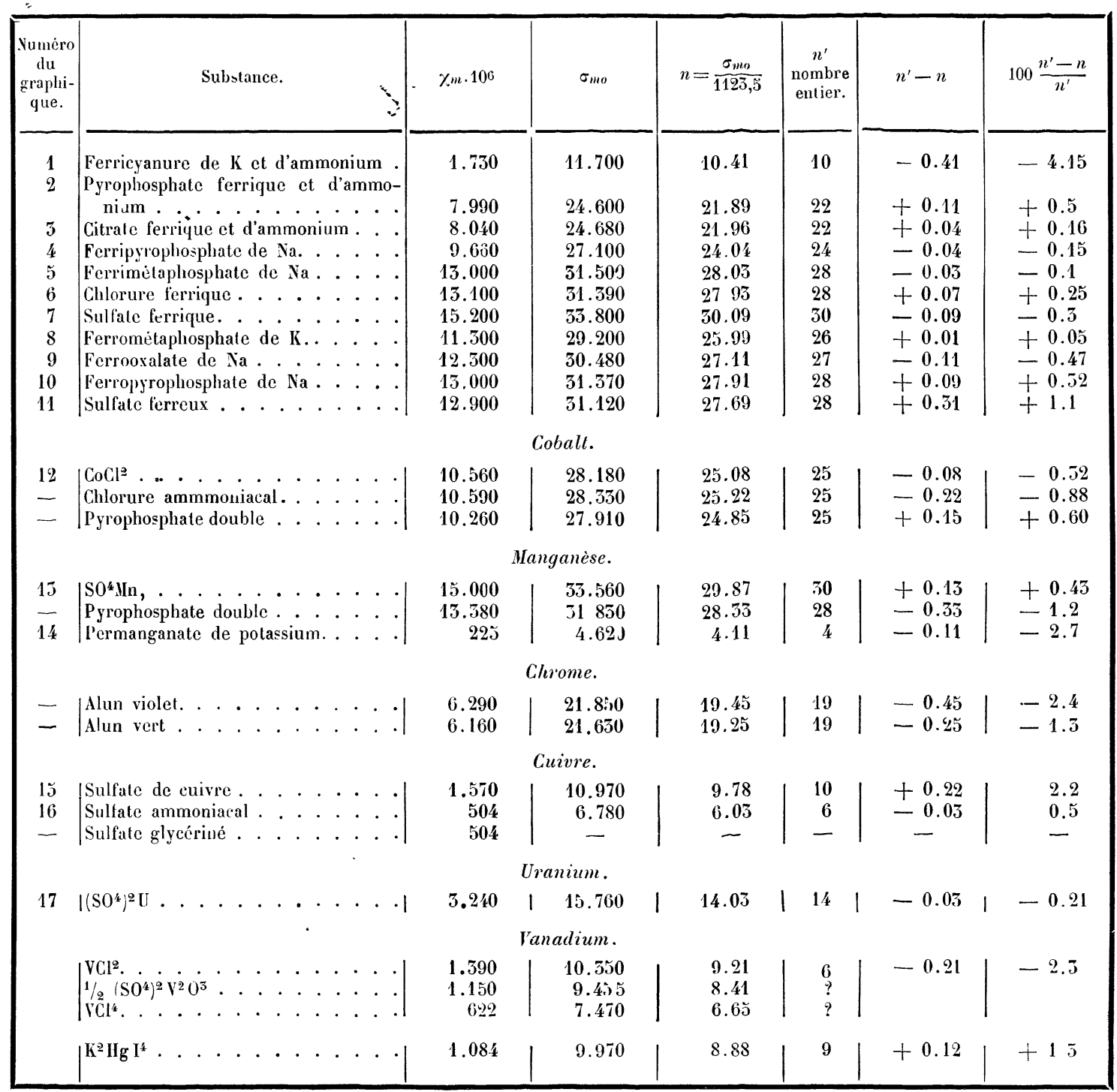

T. 8. 
antérieurement. Au travail de Pascal doit ètre rattaché celui de Liebknecht et Wills ${ }^{1}$, qui a porté sur les sels de nickel en solution aqueuse. De ces expériences N. Weiss a pu déduire qu'aux composés suivants : $\mathrm{NiF}^{2}$, $\mathrm{Ni} \mathrm{Cl}^{2}$, $\mathrm{Ni} \mathrm{Br}^{2}$, $\mathrm{NiI}^{2}$, $\mathrm{NiSO}^{4}, \mathrm{Ni}\left(\mathrm{A}_{z} \mathrm{O}^{5}\right)^{2}$, correspondent respectivement les nombres de magnétons : 16,$06 ; 16,11 ; 16,06 ; 16,01 ; 15,89 ; 16,02 ;$ soit 16 avec beaucoup d'exartitude.

Corps magnétiques à l'ètat solide. - A l'é.at solide, les substances magnétiques vérifient encore parfaitement la théorie, bien qu'alors les conditions ne soient plus aussi simples que dans les cas précédents, surtout lorsque la substance considérée est cristalliséc.

M. Urbain ${ }^{2}$ a déterminé les coefficients d’aimantation $\%_{m}$ de six mélaux appartenant aux terres rares, et il a trouvé les nombres du tableau III. magnéton-gramme, ct il a trouré 1122,7, soit à $\frac{7}{10000}$ piès, le nombre adopté.

Les résulfats ohtenus par Mlle Feỵtis', bien que moins précis que tous les précédents, sont encore nettement en faveur de l'hypothèse dı magnéton. On peut s'en assurer sur le tableau IV.

Métaux ferro-magnétiques au-dessus du point de Curie. - Des mesures récentes ont été effectućes par MII. Weiss et Fuex sur le nickel et le fer.

a) Nickel. - Jeux nombres ont élé obtenus : l'un, directement pour le nickel pur; l'autre, comme limite des ferro-nickels réversibles (tableau V).

Il est remarquable que les nombres de magnétons trouvés pour le nickel soient si différents les uns des autres, suivant les conditions dans lesquelles on les

Tableau III.

\begin{tabular}{|c|c|c|c|c|c|c|c|c|}
\hline $\begin{array}{c}\text { Numéro } \\
\text { du } \\
\text { iraphi- } \\
\text { que. }\end{array}$ & & Substancer & $\% m \cdot 10^{\prime s}$ & $\sigma_{m !}$ & $n=\frac{\sigma_{m o}}{1123 . \ddot{\partial}}$ & $\begin{array}{c}u^{\prime} \\
\text { nombre } \\
\text { entier. }\end{array}$ & $n^{\prime}-n$ & $100 \frac{n^{\prime}-n}{n^{\prime}}$ \\
\hline $\begin{array}{l}1 \\
2 \\
5 \\
1 \\
j \\
6\end{array}$ & $\begin{array}{c}\text { Se-quiourde } \\
- \\
- \\
-\end{array}$ & 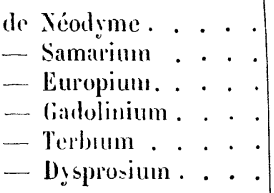 & $\begin{array}{r}5.630 \\
1.154 \\
5.893 \\
29.189 \\
43.450 \\
54.200\end{array}$ & $\begin{array}{r}20.250 \\
9.105 \\
20.700 \\
46065 \\
56.160 \\
62.650\end{array}$ & $\begin{array}{r}18.021 \\
8.01 j \\
18.598 \\
40.971 \\
49.986 \\
55790\end{array}$ & $\begin{array}{r}18 \\
8 \\
18 \\
41 \\
50 \\
56\end{array}$ & $\begin{array}{r}-0.024 \\
-0.015 \\
-0.598 \\
+0.099 \\
+0.014 \\
+0.210\end{array}$ & $\begin{array}{l}0.17 \\
0.19 \\
2.21 \\
0.07 \\
0.05 \\
0.57\end{array}$ \\
\hline
\end{tabular}

Tableau IV.

\begin{tabular}{|c|c|c|c|c|c|c|}
\hline Substances. & $\% m \cdot 10^{6}$ & $\sigma_{m}$, & $n=\frac{\sigma_{m u}}{1125 . \dot{3}}$ & $\begin{array}{c}n^{\prime} \\
\text { nombre } \\
\text { entler. }\end{array}$ & $n^{\prime}-n$ & $100 \frac{n^{\prime}-n}{n^{\prime}}$ \\
\hline $\mathrm{FeCl}^{3}$ sublimé.. . . . . . . . . . . . & 14.630 & 52.400 & 28.83 & 29 & +0.17 & +0.58 \\
\hline $\mathrm{FeCl}^{3}, 2 \mathrm{NH}^{4} \mathrm{Cl}, \mathrm{I}^{2} \mathrm{O}$. . . . . . . . . . . & 12.850 & 50.350 & 26.99 & 27 & +0.01 & +0.0 .3 \\
\hline $\mathrm{FeF}^{3}, \mathrm{SNll}^{4} \mathrm{~F} . . . . . . .$. & 14.820 & 32.660 & 28.94 & 29 & +0.06 & +0.43 \\
\hline $\mathrm{FeF}^{3}, 2 \mathrm{NII}^{4} \mathrm{~F}, \mathrm{Il}^{2} \mathrm{O} . \ldots . .$. & 14.965 & $3 ! .800$ & 29.19 & 29 & -0.19 & -0.61 \\
\hline 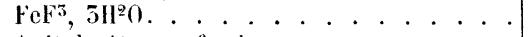 & 7.899 & 25.860 & 21.23 & 21 & -0.23 & -1.10 \\
\hline 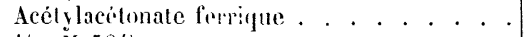 & 10.922 & $28.1: 0$ & $250 \tilde{3}$ & 25 & -0.05 & -0.21 \\
\hline 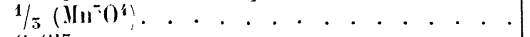 & 5.730 & 20.190 & 17.97 & 18 & +0.03 & +0.16 \\
\hline 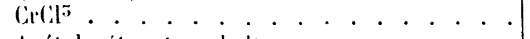 & 7.027 & 22.510 & 20.04 & 20 & -0.04 & -0.18 \\
\hline Icétylacélonale coballeus. . . . . . . . . & 7.767 & 23.730 & 21.12 & 21 & -0.12 & -0 ถั \\
\hline $1 / 2\left[\mathrm{Cr}\left(\mathrm{MI}^{3}\right)^{6}\right]\left[\mathrm{Cr}^{\prime}\left(\mathrm{L}^{3} \mathrm{O}^{4}\right)^{3}\right]+3 \mathrm{H}^{2} \mathrm{O}$ & 14.160 & 22.6 .00 & 20.16 & 20 & -0.16 & -0.80 \\
\hline 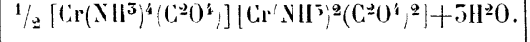 & 14.160 & 22.6 .00 & 20.16 & 20 & -0.16 & -0.80 \\
\hline
\end{tabular}

Tableau V.

\begin{tabular}{|c|c|c|c|c|c|c|c|}
\hline Sulistunce's. & C & $\mathrm{C}_{m}$ & $\sigma_{m o}$ & $n=\frac{\sigma_{m n}}{1123,3}$ & $\begin{array}{c}n^{\prime} \\
\text { nombre } \\
\text { enticr. }\end{array}$ & $n^{\prime}-n$ & $100 \frac{n^{\prime}--n}{n^{\prime}}$ \\
\hline $\begin{array}{l}\text { Nichel pur directement . . } \\
\text { Limite des ferro-nickels. . }\end{array}$ & $\begin{array}{l}0.00505 \\
0.00700\end{array}$ & $\begin{array}{l}0.5258 \\
0.4109\end{array}$ & $\begin{array}{r}9.018 \\
10.130\end{array}$ & $\begin{array}{l}8.03 \\
9.05\end{array}$ & $\begin{array}{l}8 \\
9\end{array}$ & $\begin{array}{l}-0.03 \\
-0.05\end{array}$ & $\begin{array}{l}-0.28 \\
-0.27\end{array}$ \\
\hline
\end{tabular}

(11 voit qu'en dehors du dysprosium et de l'europium, les résultats sont excellents. Des observations $1,2, \dot{4}, 5,6, \mathbf{6}$. Weiss a encore déduit la valeur du

\footnotetext{
1. Litbikecht et Wills, Aun. d. Phys., (1900) 178

2. Јивмк. .. R.. $147(1908 ; 1286$.
}

détermine (on a lu suceessivement les nombres suivants : 5, 16, 8, 9). Peut-être est-ce lì une indicatıon d'une inlluence du milieu, ayant un retenlissement sur la structure intime de l'atome.

1. E. Ferres. (C. Ri. 152 1911) 70x. 
b) Fer. - Le cas du fer, beaucoup plus complexe, conduit à des résultats très intéressants. En conservant l'hypothèse faite jusqu'à présent à savoir : deux degrés de lib rté de rotation par alome, on trouve les numbres ci-dessous : (tableau VI).

On voit de suite que les valeurs de $n$ s'écartent des entiers les plus proches beaucoup plus que ne le per- l'un à l'autre, on trouve 13,02 magnétons pour chacun d'eux, ce qui est un nombre entier avec toute la précision désirable.

\section{Gonclusions.}

En résumé, nous avons vu que le's moments magnéTableau VI.

\begin{tabular}{|c|c|c|c|c|c|}
\hline Substances. & $\begin{array}{ll}\text { Intervalle } \\
\text { de } \\
\text { température. }\end{array}$ & $\mathrm{C}$ & $\mathrm{C}_{m}$ & $\sigma_{m o}$ & $n=\frac{\sigma_{m o}}{1120, \tilde{0}, \ddot{3}}$ \\
\hline $\begin{array}{l}\text { Fer } \beta_{1} \ldots \ldots . \\
\text { Fer } \beta_{2} . \\
\text { Fer } \gamma .\end{array}$ & $\begin{array}{l}774^{0}-828^{\circ} \\
828^{\circ}-920^{\circ} \\
920^{\circ}-1395^{\circ}\end{array}$ & $\begin{array}{l}0.0395 \\
0.0273 \\
0.072\end{array}$ & $\begin{array}{l}2.213 \\
1.529 \\
4.03\end{array}$ & $\begin{array}{l}23.500 \\
19 . \check{3} 30 \\
511730\end{array}$ & $\begin{array}{l}20.92 \\
17.38 \\
28.25\end{array}$ \\
\hline
\end{tabular}

mettraient les erreurs d'expérience. M. Weiss a róussi à meltre ces résultats d'accord avec la théorie du magnéton en imaginant l'hypothise suivante: le ler $\beta$ serait constitué par une molécule rigide $\mathrm{Fe}^{\bar{s}}$, le ler $\gamma$ par une molécule rigide $\mathrm{Fe}^{2}$ : le fer ò par unc molécule $\mathrm{Fc}$. I)ans cette manière de voir, le tableau précédent se transforme dans le suivant: tiques d'un même atome lorsqu'il ('st dans les conditions les plus variées, ou bien d'atomes différents, sont des multiples entiers d'un moment magnétique élémentaire, le magnéton. La démonstration est faite actuellement pour les atomes de $\mathrm{Fe}, \mathrm{Ni}, \mathrm{Co}, \mathrm{Cr}, \mathrm{Mn}$, $\mathrm{I}, \mathrm{Cu}, \mathrm{Hg}, \mathrm{U}$, et ceux des métaux des terres rares.

M. Weiss élablit un rapprochement très curieux

Takleau VIr.

\begin{tabular}{|c|c|c|c|c|c|c|c|}
\hline Sulislances. & C & $\mathrm{C}_{m}$ & $\begin{array}{c}\sigma_{a 0} \\
\text { par atome. }\end{array}$ & $n=\frac{\sigma_{a^{\prime \prime}}}{112 \bar{\jmath}, \ddot{0}}$ & $\begin{array}{c}n^{\prime} \\
\text { nombre } \\
\text { entier. }\end{array}$ & $n^{\prime}-n$ & $\frac{n^{\prime}-n}{n^{\prime}}$ \\
\hline 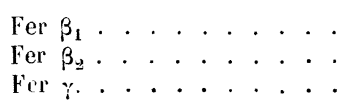 & $\begin{array}{l}0.0595 \\
00273 \\
0.072\end{array}$ & $\begin{array}{l}5 \times 2.213 \\
5 \times 1.529 \\
2 \times 4.03\end{array}$ & $\begin{array}{l}15.567 \\
11.277 \\
22.420\end{array}$ & $\begin{array}{l}12.18 \\
10.04 \\
19.95\end{array}$ & $\begin{array}{l}12 \\
10 \\
20\end{array}$ & $\begin{array}{r}008 \\
-0.04 \\
+\quad 0.05\end{array}$ & $\begin{array}{l}-0.7 \\
-0.4 \\
+0.25\end{array}$ \\
\hline
\end{tabular}

Ce:te fois, les valeurs de $n$ sont parfaitement acceptab'es; il ne manque, à une vérificalion complète de cette dernière hypothèse, que les nombres relatifs au fer ò : ce dernier n’a pu être étudié que très peu, en raison des grandes difficultés expérimentales que l'on rencontre yuand on veut faire des mesures à des températures supérieures à $1400^{\circ}$.

Il est possible peut-être d'expliquer au moyen d'une hypothèse analogue, le désaccord apparent observé cidessus dans le cas de l'europium : en admettant que deux alomes de ce dernier sont reliés rigidement entre lihypothèse du magnéton et la théorie de Ritz concernant les lois des spectres en série; on sait que ce dernier a imaginé, pour expliquer la loi de Balmer, ou les lois voisines, un mécanisme électro-magnélique, qui consiste essentiellement en bâtonnets de moments magnétiques donnés identiques entre eux et disposés d'une manière rigide en piles linéaires; peut-être a-t-on li le magnéton mis en évidence au courant du présent travail?

[Evtrails par J. D.1,rs] [lieçu le jooù 1911]. 